\title{
WNT5A Correlates with Clinicopathological Characteristics in Gastric Cancer: a Meta- Analysis
}

\author{
Seungyoon Namª, ${ }^{a, b}$ Jun-Won Chung ${ }^{d}$ Jun-Young Yang ${ }^{e}$ \\ aDepartment of Genome Medicine and Science, College of Medicine, Gachon University, Incheon, \\ ${ }^{b}$ Department of Life Sciences, Gachon University, Seongnam, 'Gachon Institute of Genome Medicine \\ and Science, Gachon University Gil Medical Center, Incheon, 'Division of Gastroenterology, Department \\ of Internal Medicine, Gil Medical Center, Gachon University College of Medicine, Incheon, eDepartment \\ of Surgery, Gil Medical Center, Gachon University College of Medicine, Incheon, Korea
}

\section{Key Words}

WNT5A • Gastric cancer $•$ Lymph node metastasis $•$ Meta-analysis

\begin{abstract}
Background/Aims: Gastric cancer (GC), the third-leading cause of cancer death in the world, is typically diagnosed only in its advanced stages. WNT signaling has been associated with clinicopathological characteristics in diverse cancer types. But the systematic analysis of WNT5A, a member in the signaling, has not been inspected. Thus, our study used a metaanalysis to statistically associate WNT5A expression with GC clinicopathological characteristics. Methods: For a systematic literature review of GC in combination with the WNT signaling molecule WNT5A, we searched for PubMed, Cochrane Library, and Web of Science. It led to the five cohorts, in four eligible studies, consisting of 1,034 patients (617 WNT5A-positive and 417 WNT5A-negative patients). These patients were inspected by the library "meta" in R software for our meta-analysis. Results: Our meta-analysis, revealed a statistically significant associations of WNT5A-positivity with lymph node metastasis $(p=0.0047)$, some types of Lauren diffuse subtype GCs $(p<0.0001)$, advanced tumor depth $(p<0.0001)$, and advanced UICC stages $(p=0.0461)$ with no observation of bias or confounding factors. Conclusions: These results support the feasibility of targeting this embryonic signaling pathway, both for therapy, and as a biomarker to "guide" various individual interventions (i.e., "personalized medicine").

\section{Introduction}

Gastric cancer (GC) had 951,600 new cases, worldwide, and 723,100 deaths in 2012 [1]. The disease is more prevalent in East Asia (including Korea, Japan, and China) than in other regions. However, effective targeted therapies and clinically available GC biomarkers have yet to be developed [2-5]. 
WNT5A, a member of the embryonic signaling pathway Wingless (WNT), is 379 amino acids (a.a) in length, with several richly conserved cysteines [6]. WNT5A, a ligand for members of the Frizzled family of seven transmembrane receptors, plays critical roles in postnatal cellular functions, as well as development through WNT signal pathways [6]. Also, WNT5A has been involved in diverse pathogeneses, including cancers, metabolic diseases, and inflammatory diseases, through multiple WNT signaling receptors [6]. In cancer, diverse studies of WNT5A show that its expression associates with clinicopathological characteristics [7]. Also, our previous study reported WNT5A expression associated with the Lauren classification of GC [8].

Considering the aforementioned increasing importance of WNT5A, in terms of both functions and clinical implications, we embarked upon a systematic inspection of associations between WNT5A expression and clinicopathological characteristics. By collecting multiple publications systematically and increasing the sample size of GC patients, we performed a meta-analysis, enabling us to evaluate the clinical relevance of WNT5A expression in GC.

\section{Materials and Methods}

\section{Literature inspection}

To identify clinically relevant publications regarding WNT5A expression, we searched PubMed, Cochrane library, and Web of Science for literature reports through $3^{\text {rd }}$ Sep 2016, with the following terms: "WNT5A" and "gastric" and "cancer". We obtained all publications resulting from this search, of which titles and abstracts included relevant information for our meta-analysis (Fig 1).

\section{Eligibility Criteria}

Eligible articles in our meta-analysis satisfied the following conditions: i) literature citations related to gastric cancer and WNT5A expression; ii) the patients were pathologically diagnosed with gastric cancer; iii) requirement of a case-control design; and iv) enough data to obtain odds ratios (ORs) and $95 \%$ confidence intervals (CIs).

\section{Exclusion Criteria}

The following types of literature reports were excluded: i) reviews; ii) case reports; iii) studies reporting incorrect numbers of patients having WNT5A positivity; iv) studies of other cancer types; v) studies lacking information of the number of WNT5A-positive and -negative patients; and vi) studies dealing with DNA methylation and epigenetics of WNT5A in GC patients.

\section{Data Extraction}

The two author reviewers (SN, JJ) independently reviewed eligible literature citings, satisfying the two aforementioned criteria, to extract relevant information for our meta-analysis. This information included authors, publication year, study objects, WNT5A positivity criteria, method, ethnicity, and clinical parameters.

\section{Statistical Analysis}

To see statistical association between WNT5A expression and GC clinical clinicopathological factors, pooled ORs and 95\% CIs were calculated. The clinicopathological factors considered tumor depth, lymph node metastasis, Lauren histological classification, and UICC (Union for International Cancer Control) TNM classification. Lauren classification was divided by "diffuse type versus
Fig. 1. Flow of systematic publication selection processes.

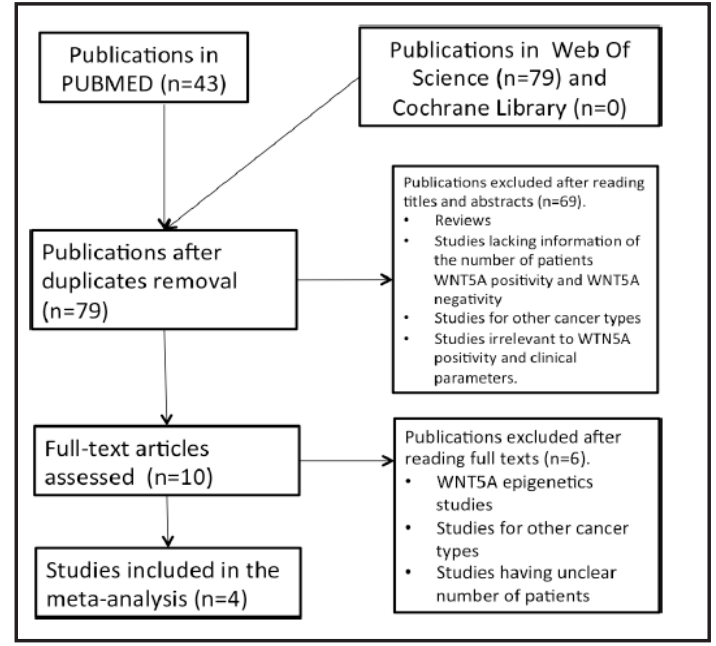




\section{Cellular Physiology Cell Physiol Biochem 2017;41:33-40

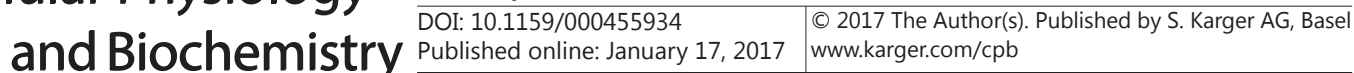 \\ Nam/Chung/Yang: A Meta-Analysis of WNT5A in Gastric Cancer}

intestinal type" or "diffuse-scattered type versus diffuse-adherent type plus intestinal type". UICC stages were divided to "I/II versus III/IV" or "I versus II/III/IV".

We used the library "meta" [9], in R software (www.r-project.org), to perform the meta-analysis, heterogeneity statistics $\left(T^{2}, I^{2}\right)$ (including Q-tests) between studies, publication bias, and sensitivity analysis. The heterogeneity was measured by Q-tests and their p-values. Considering that $I^{2} \leq 50 \%$ or p-value $<0.05$ indicates the absence of heterogeneity [10], we applied fixed effect models to obtain pooled ORs. Otherwise, we used random-effect models to calculate pooled ORs $[10,11]$. Publication biases were visually inspected by funnel plots (standard error of OR vs. OR), and all plots showed symmetry, indicating no deviation among publications. In sensitivity analysis, the effect of each study on pooled ORs was inspected by omitting a study one by one.

\section{Results}

\section{Included studies and their information}

Forty-three studies were obtained from PUBMED, and seventy-nine studies from other sources. After careful review of the studies through their titles and abstracts, fortythree studies were duplicated, and removed, and sixty-nine studies were removed due to irrelevance. The resultant ten studies were carefully reviewed by reading their full-texts, and four studies were removed due to WNT5A epigenetics studies, studies for other cancer types, and studies having unclear number of patients. As a result, four publications (Table 1) were selected for meta-analysis. One study [8] had two independent cohorts, and a total of five cohorts/datasets were eligible for our meta-analysis (Table 1). The selection processes are depicted in Fig 1. Expression of WNT5A was measured through immunohistochemistry (IHC).

\section{Statistical associations between WNT5A expression, and clinicopathological parameters,} in $G C$

Five datasets involving 1,034 patients having 617 WNT5A-positive GC patients and 417 WNT5A-negative GC patients showed positive correlation of Wnt5 expression with lymph node metastasis (OR (N1 N3 versus $\mathrm{N} 0)=3.01 ; 95 \% \mathrm{CI}=1.40 \sim 6.45 ; \mathrm{P}=0.0047$; randomeffect; Fig 2A). Furthermore, WNT5A positively associated with UICC stage (OR (III IV versus $\mathrm{I} \sim \mathrm{II})=2.79 ; 95 \% \mathrm{CI}=1.02 \sim 7.66 ; \mathrm{P}=0.0461$; random-effect; Fig $2 \mathrm{~B})$. Wnt5 expression also positively associated with tumor depth T stages T2 T4 versus T1 (OR (T2 T4 versus $\mathrm{T} 1)=8.35 ;$ 95\% CI=3.91 17.83; P < 0.0001; fixed-effect; Fig 2C). WNT5A expression significantly associated with "Lauren diffused scattered" (indicated in DS in Fig 2D) subtype versus "Lauren diffuse adherent subtype plus Lauren intestinal subtype (indicated in Others in Fig 2D)" (OR (DS versus Others) $=6.69$; 95\% CI=4.00 11.18; $\mathrm{P}<0.0001$; fixed-effect; Fig 2D). However, WNT5A expression was not statistically associated with tumor depth stages $\mathrm{T} 3 \sim \mathrm{T} 4$ versus $\mathrm{T} 1 \sim \mathrm{T} 2(\mathrm{P}=0.8001$; Fig $2 \mathrm{E})$; sex $(\mathrm{P}=0.1396$; Fig $1 \mathrm{~F})$; or Lauren subtypes diffuse versus intestinal $(\mathrm{P}=0.4663$; Fig $2 \mathrm{G})$.

Table 1. Information of cohorts eligible for our meta-analysis of WNT5A in gastric cancer. Chang et al. [8] previously reported two independent cohorts: one for discovery (henceforth, Chang_D), and the other for validation (henceforth; Chang_V). In our meta-analysis, we used the two cohorts separately. So, the four selected publications had the five cohorts (datasets). NA: not available

\begin{tabular}{|c|c|c|c|c|c|c|c|c|}
\hline $\begin{array}{l}\text { Author } \\
\text { (dataset) }\end{array}$ & $\begin{array}{l}\text { Publication } \\
\text { year }\end{array}$ & Ethnicity & Method & $\begin{array}{l}\text { WNT5A- } \\
\text { positive }\end{array}$ & $\begin{array}{l}\text { WNT5A- } \\
\text { negative }\end{array}$ & Dilution & Positivity criteria & $\begin{array}{l}\text { WNT5A } \\
\text { antibody }\end{array}$ \\
\hline Chang et al. [8] (Chang_D) & 2016 & Korean & $\mathrm{IHC}$ & 195 & 56 & $1: 5000$ & Grading score $\geq 1$ & Abgent \\
\hline Chang et al. [8] (Chang_V) & 2016 & Korean & $\mathrm{IHC}$ & 214 & 37 & $1: 5000$ & Grading score $\geq 1$ & Abgent \\
\hline $\begin{array}{l}\text { Kanzawa et al. [19] } \\
\text { (Kanzawa) }\end{array}$ & 2013 & Japanese & IHC & 93 & 49 & NA & $\begin{array}{l}\text { Over } 50 \% \text { of cancer cells indicating moderate to } \\
\text { intense immunoreactivity }\end{array}$ & Abcam \\
\hline $\begin{array}{l}\text { Yamamoto et al. [24] } \\
\text { (Yamamoto) }\end{array}$ & 2009 & Japanese & $\mathrm{IHC}$ & 44 & 109 & NA & More than $50 \%$ of cancer cells were stained. & NA \\
\hline $\begin{array}{l}\text { Kyurayoshi et al. } \\
\text { [18](Kyurayoshi) }\end{array}$ & 2006 & Japanese & $\mathrm{IHC}$ & 71 & 166 & NA & More than $50 \%$ of cancer cells were stained. & In-house \\
\hline
\end{tabular}


Fig. 2. Meta-analysis results of clinical parameters. The first column indicates study names; the second column experimental group; the third column control group; the forth column forest plot; the fifth column OR of the experimental group versus the control group; the sixth column 95\% CI; and the seventh column weight (indicated in W). (A) Lymph node metastasis $\mathrm{N} 1 \sim \mathrm{N} 3$ versus no regional metastasis N0. (B) UICC stages III IV versus I II. (C) Tumor depth T stages T2 T4 versus T1. (D) Lauren diffuse-scattered (DS) subtype versus "Lauren diffuse-adherent subtype plus Lauren intestinal subtype" (Others). (E) Tumor depth stages T3 T4 versus $\mathrm{T} 1 \sim \mathrm{T} 2$. (F) Sex male versus female. $(G)$ Lauren subtypes diffuse versus intestinal.

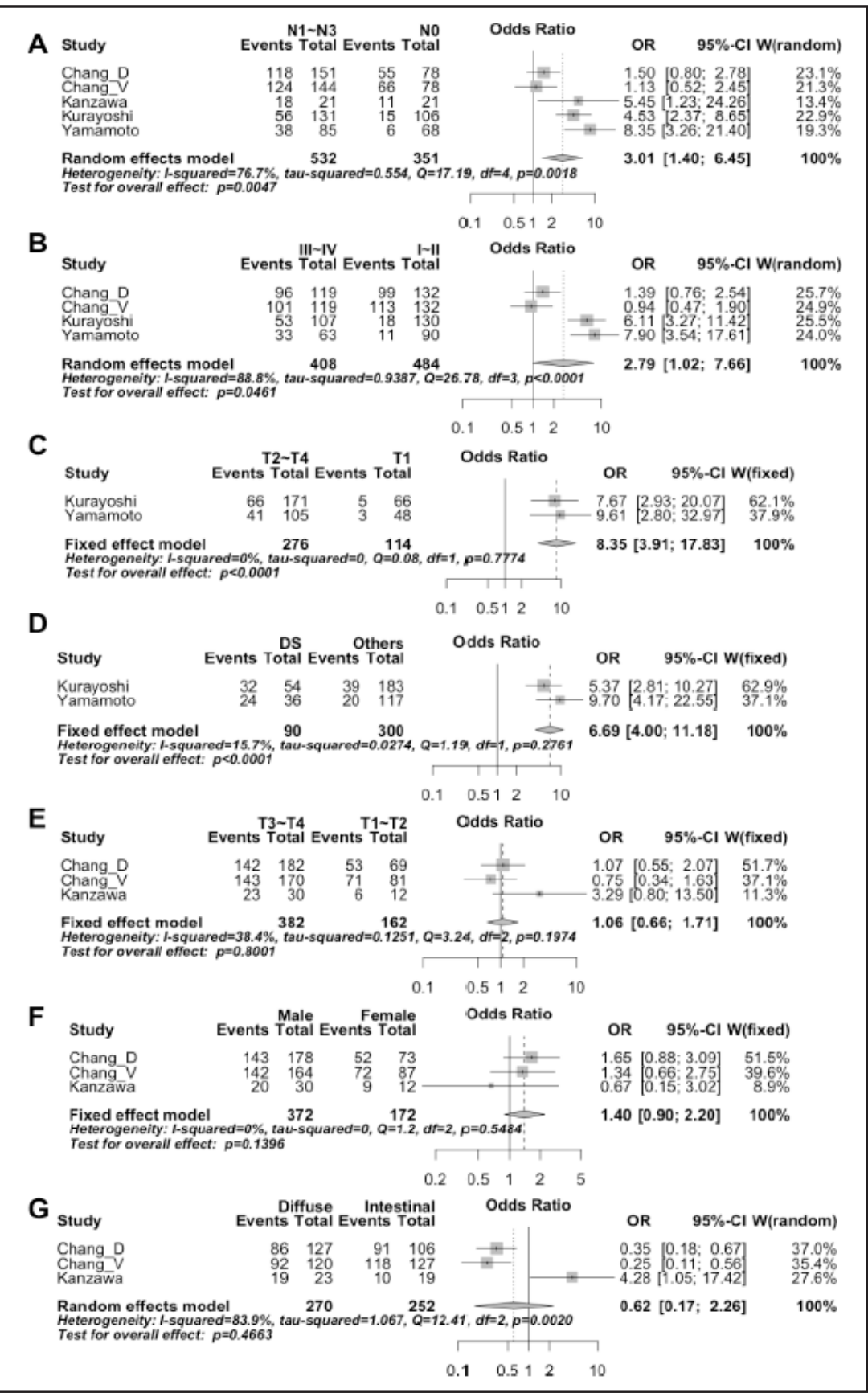

Sensitivity analysis and publication bias

To measure effects of individual studies on the overall conclusions, we performed sensitivity analysis by removing each study one-by-one. The sensitivity analysis indicated that individual studies did not affect the conclusions (Fig 3). The ORs (in the previous section) of tumor depth stages (T2 T4 vs. T1) (Fig 3D), lymph node metastasis (N1 N3 vs. N0) (Fig 3E), Lauren subtypes (Lauren diffused scattered subtype vs. "Lauren diffuse adherent subtype plus Lauren intestinal subtype") (Fig 3C), and UICC stages (III IV vs. I II) (Fig 3G) were not changed in some contexts, even though individual studies were omitted, thus reinforcing the robustness of the conclusions.

We next inspected publication biases for the statistically significant associations shown in Fig 2, visually confirming no asymmetries (Fig 4). Thus, it indicated no publication biases for the significant associations. 


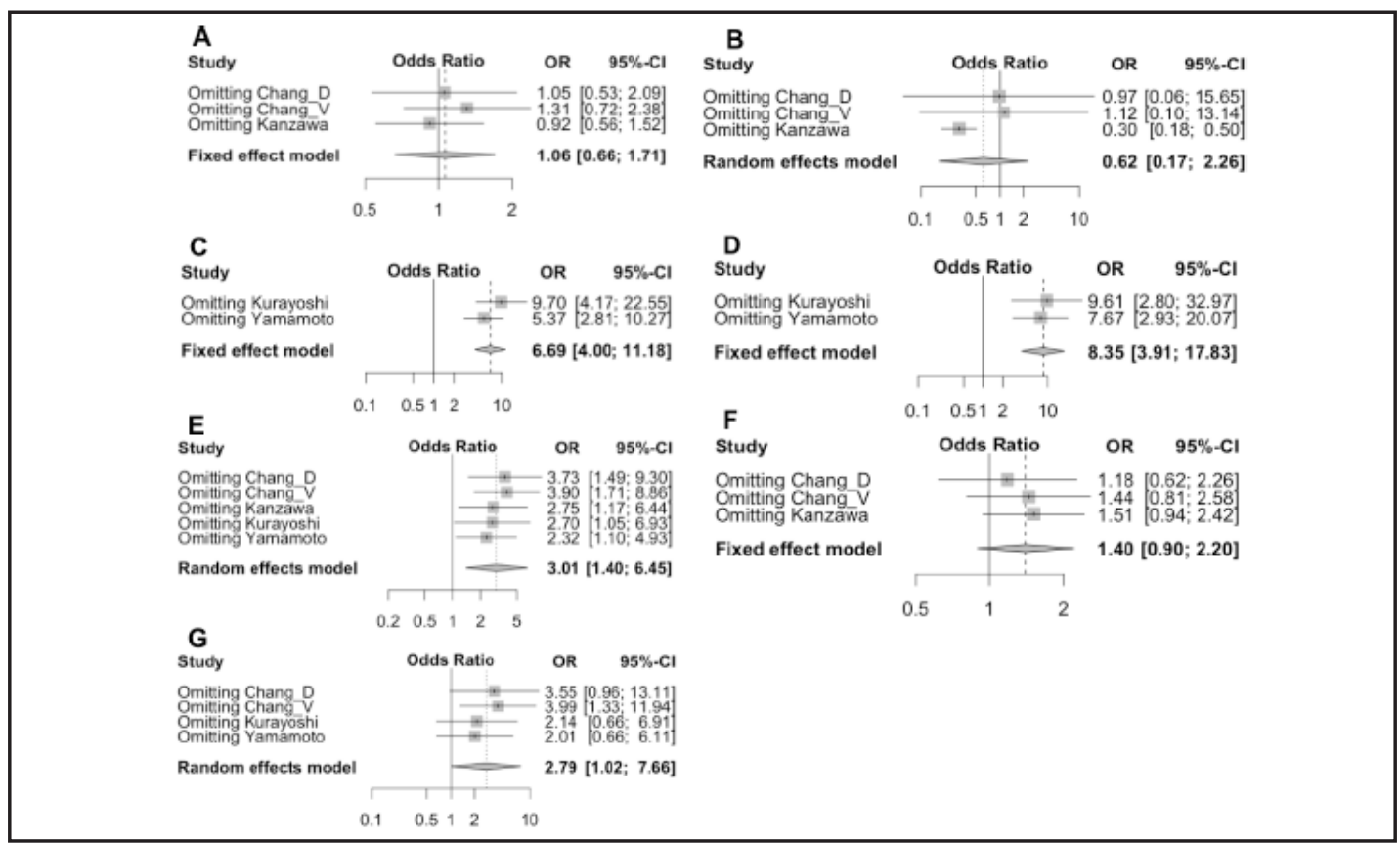

Fig. 3. Sensitivity analyses of the meta-analysis results. (A) Tumor depth stages T3 T4 versus T1 T2. (B) Lauren subtypes diffuse versus intestinal. (C) Lauren diffuse-scattered (DS) subtype versus "Lauren diffuseadherent subtype plus Lauren intestinal subtype" (Others). (D) Tumor depth T stages T2 T4 versus T1. (E) Lymph node metastasis N1 N3 versus no regional metastasis N0. (F) Sex male versus female. (G) UICC stages III $\sim$ IV versus I II.

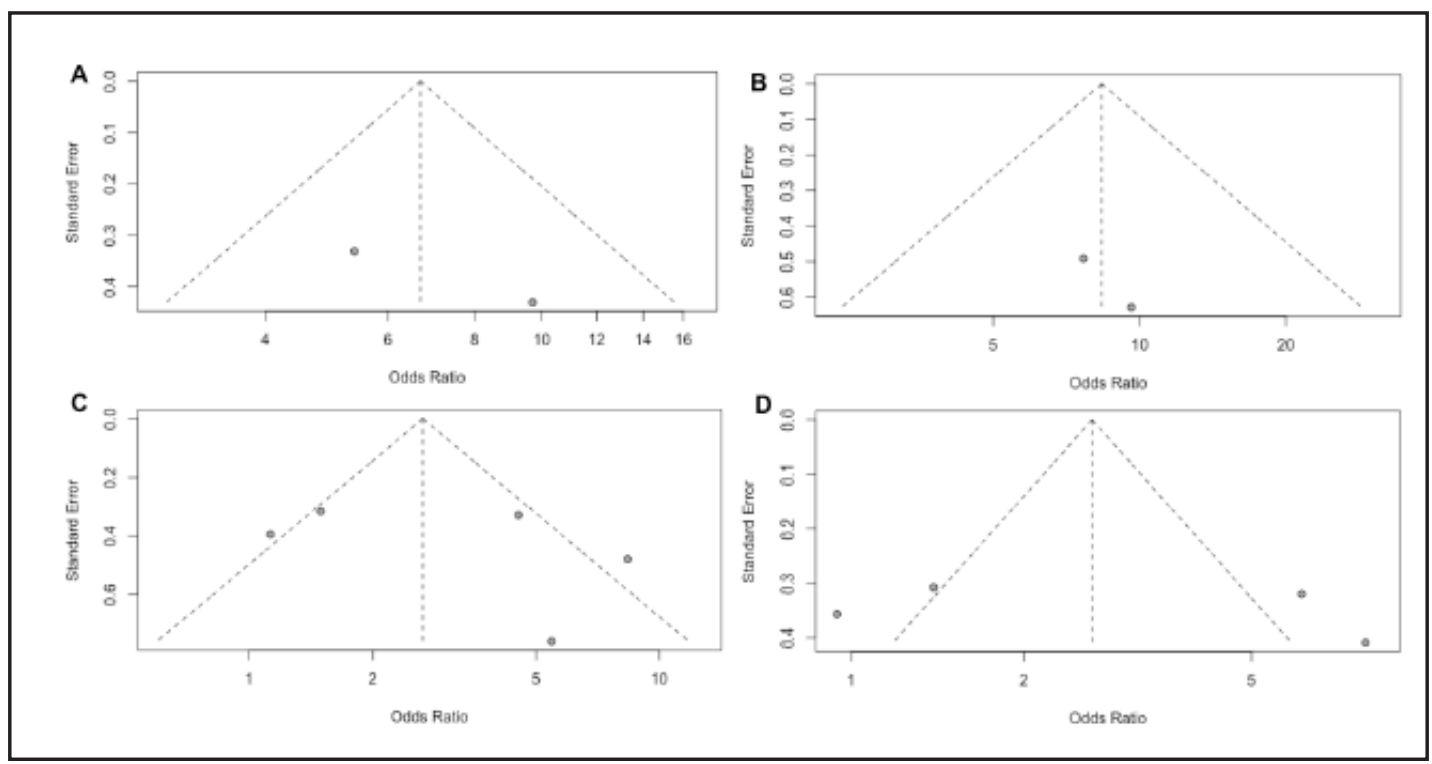

Fig. 4. Funnel plots for inspecting publication biases graphically. We inspected publication biases for the significant associations (of Fig 2). (A) Lauren diffuse-scattered (DS) subtype versus "Lauren diffuseadherent subtype plus Lauren intestinal subtype" (Others). (B) Tumor depth T stages T2 T4 versus T1. (C) Lymph node metastasis N1 N3 versus no regional metastasis N0. (D) UICC stages III IV versus I II.

\section{Discussion}

GC is one of the most common cancer deaths in East Asia, including Korea, China and Japan [1], and globally, is the third-leading cause of cancer death [12]. Recently, two large- 


\section{Cellular Physiology Cell Physiol Biochem 2017;41:33-40

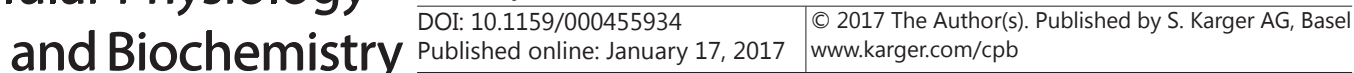 \\ Nam/Chung/Yang: A Meta-Analysis of WNT5A in Gastric Cancer}

scale GC studies $[13,14]$ of next-generation sequencing demonstrated multiple molecular subtypes could feasibly be used for patient stratification for potential GC therapies. Of interest, Cristescu et al. [13] reported a statistically significant association between Lauren diffuse subtype GC and a molecular subtype MSS/EMT (microsatellite stable/epithelialto-mesenchymal transition). Despite progress in the molecular understanding of GC, this neoplasm still lacks clinically available biomarkers, as well as established targeted therapies [4], largely due to the nature of GC heterogeneity. Consequently, despite recent progress in the field of HER2-positive advanced GC [2], the direct benefit to GC patients remains limited. Despite the many efforts to identify GC clinically relevant biomarkers, the lack of feasible specifying patient groups for stratification, potential targeted therapies continue to be sought. Consequently, we herein performed a systematic review for GC clinical relevance through multiple publications, since WNT5A (a member of WNT signaling [15]) has been well-implicated in GC progression, and possible druggable signaling, in our previous studies $[8,16]$.

In the present study, we confirmed that WNT5A positivity was statistically relevant to clinical parameters such as lymph node metastasis, UICC stage progression, a certain subtype of Lauren diffuse GC, and increased tumor depth. These findings strongly suggest that WNT signaling is a clinically valuable tool as a GC biomarker, and possible target for therapy.

Functionally, WNT signaling $[15,17]$ has been implicated in several types of cancer. WNT5A targeting in GC cell lines and xenograft models demonstrated antitumor activity, supporting its value as a potential therapeutic target in GC [16]. Also, HNF4 $\alpha$ (hepatic nuclear factor-4) is an upstream regulator of WNT5A transcription, and we have shown the HN4 $\alpha$ WNT5A axis to be inhibited by small molecular weight compounds, in vitro, and xenograft models [8]. We also showed that WNT5A was involved in cell migration in GC cells through focal adhesion pathway and the Rac1 protein [18]. These computational cellular functions in GC cells closely align with our current meta-analysis results, indicating positive association between lymph node metastasis and WNT5A-positivity in GC patients. Recently, WNT5A [19] has been implicating, in GC cells, in inducing the epithelial-mesenchymal transition (EMT), a complex phenomenon involving drastic changes in cellular morphology and various phenotypes [20], largely inhibiting differentiation-related genes. Thus, the association between WNT5A-positivity and advanced cancer stages, in our meta-analysis, may result from WNT5A-driven EMT, resulting in metastasis and other advanced tumor phenotypes.

Considering the aforementioned functional roles of WNT5A, as well as our metaanalysis results, WNT signaling is critical for two clinical aspects: 1) potential biomarkers; and 2) therapeutic target candidates. WNT signaling regulation by compounds in cancer has not been successful, due to safety concerns, although those concerns are also applicable to other drugs [17]. Dissecting GC patients based on an evidence-based approach may increase the possibility of WNT signaling as a druggable target, and meta-analysis can provide clues for WNT5A's use for patient stratification, and as a potential "druggable" target for small compounds.

The significance of tumor depth stages (Fig 2C) and Lauren subtypes (Fig 2D) should be carefully interpreted, due to the small number of publications. As WNT5A-relating studies increase in the future, another systematic review needs to be designed. Recently, GC systematic reviews have been applied to noncoding RNAs [21-23] for potential GC patient stratification.

\section{Conclusion}

We applied a meta-analysis for exploring the clinical relevance of WNT5A in gastric cancer. This work indicated that WNT5A-expression positively associates with lymph node metastasis, tumor depth, advanced stages, and a diffuse subtype. These findings support our hypothesis of WNT5A not only representing a strong candidate biomarker, but also as a therapeutic strategy.

\section{KARGER}




\section{Cellular Physiology Cell Physiol Biochem 2017;41:33-40

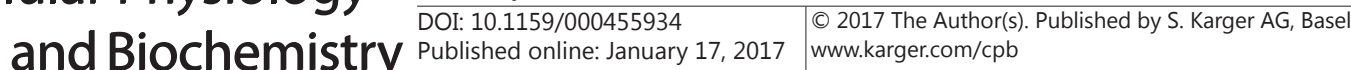

Nam/Chung/Yang: A Meta-Analysis of WNT5A in Gastric Cancer

\section{Acknowledgements}

This research was supported by Basic Science Research Program through the National Research Foundation of Korea (NRF) funded by the Ministry of Education (2016R1D1A1B03933145) (to S.N.); and the Gachon University Gil Medical Center (Grant number: 2016-06) (to S.N.). Authors thank Curt Balch for editing the draft.

\section{Disclosure Statement}

The authors declare no potential conflicts of interest.

\section{References}

1 Torre LA, Bray F, Siegel RL, Ferlay J, Lortet-Tieulent J, Jemal A: Global cancer statistics, 2012. CA Cancer J Clin 2015;65:87-108.

- Bang YJ, Van Cutsem E, Feyereislova A, Chung HC, Shen L, Sawaki A, Lordick F, Ohtsu A, Omuro Y, Satoh T, Aprile G, Kulikov E, Hill J, Lehle M, Ruschoff J, Kang YK, To GATI: Trastuzumab in combination with chemotherapy versus chemotherapy alone for treatment of HER2-positive advanced gastric or gastro-oesophageal junction cancer (ToGA): a phase 3, open-label, randomised controlled trial. Lancet 2010;376:687-697.

-3 Gao W, Xua J, Wang F, Zhang L, Peng R, Zhu Y, Tang Q, Wu J: Mitochondrial Proteomics Approach Reveals Voltage-Dependent Anion Channel 1 (VDAC1) as a Potential Biomarker of Gastric Cancer. Cell Physiol Biochem 2015;37:2339-2354.

-4 Chang HR, Park HS, Ahn YZ, Nam S, Jung HR, Park S, Lee SJ, Balch C, Powis G, Ku JL, Kim YH: Improving gastric cancer preclinical studies using diverse in vitro and in vivo model systems. BMC Cancer 2016;16:200.

-5 Niu L, Qin HZ, Xi HQ, Wei B, Xia SY, Chen L: RNF43 Inhibits Cancer Cell Proliferation and Could be a Potential Prognostic Factor for Human Gastric Carcinoma. Cell Physiol Biochem 2015;36:1835-1846.

-6 Kikuchi A, Yamamoto H, Sato A, Matsumoto S: Wnt5a: its signalling, functions and implication in diseases. Acta Physiol (Oxf) 2012;204:17-33.

-7 Gencer S, Sen G, Dogusoy G, Belli AK, Paksoy M, Yazicioglu MB: beta-Catenin-independent noncanonical Wnt pathway might be induced in gastric cancers. Turk J Gastroenterol 2010;21:224-230.

-8 Chang HR, Nam S, Kook MC, Kim KT, Liu X, Yao H, Jung HR, Lemos R, Jr., Seo HH, Park HS, Gim Y, Hong D, Huh I, Kim YW, Tan D, Liu CG, Powis G, Park T, Liang H, Kim YH: HNF4alpha is a therapeutic target that links AMPK to WNT signalling in early-stage gastric cancer. Gut 2016;65:19-32.

-9 Schwarzer G, Carpenter JR, Rücker G: Meta-analysis with R, ed 1st. Springer, 2015.

10 Pan Z, Jing W, He K, Zhang L, Long X: SATB1 is Correlated with Progression and Metastasis of Breast Cancers: A Meta-Analysis. Cell Physiol Biochem 2016;38:1975-1983.

11 DerSimonian R, Laird N: Meta-analysis in clinical trials. Control Clin Trials 1986;7:177-188.

12 GBD 2015 Mortality and Causes of DeathCollaborators: Global, regional, and national life expectancy, all-cause mortality, and cause-specific mortality for 249 causes of death, 1980-2015: a systematic analysis for the Global Burden of Disease Study 2015. Lancet 2016;388:1459-1544.

-13 Cristescu R, Lee J, Nebozhyn M, Kim KM, Ting JC, Wong SS, Liu J, Yue YG, Wang J, Yu K, Ye XS, Do IG, Liu S, Gong L, Fu J, Jin JG, Choi MG, Sohn TS, Lee JH, Bae JM, Kim ST, Park SH, Sohn I, Jung SH, Tan P, Chen R, Hardwick J, Kang WK, Ayers M, Hongyue D, Reinhard C, Loboda A, Kim S, Aggarwal A: Molecular analysis of gastric cancer identifies subtypes associated with distinct clinical outcomes. Nat Med 2015;21:449-456.

14 Cancer Genome Atlas Research Network: Comprehensive molecular characterization of gastric adenocarcinoma. Nature 2014;513:202-209.

15 Niehrs C: The complex world of WNT receptor signalling. Nat Rev Mol Cell Biol 2012;13:767-779.

-16 Nam S, Chang HR, Kim KT, Kook MC, Hong D, Kwon CH, Jung HR, Park HS, Powis G, Liang H, Park T, Kim YH: PATHOME: an algorithm for accurately detecting differentially expressed subpathways. Oncogene 2014;33:4941-4951.

17 Polakis P: Drugging Wnt signalling in cancer. EMBO J 2012;31:2737-2746. 


\section{Cellular Physiology Cell Physiol Biochem 2017;41:33-40

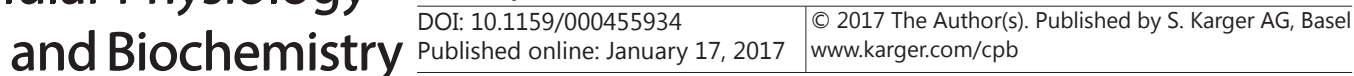 \\ Nam/Chung/Yang: A Meta-Analysis of WNT5A in Gastric Cancer}

-18 Kurayoshi M, Oue N, Yamamoto H, Kishida M, Inoue A, Asahara T, Yasui W, Kikuchi A: Expression of Wnt$5 \mathrm{a}$ is correlated with aggressiveness of gastric cancer by stimulating cell migration and invasion. Cancer Res 2006;66:10439-10448.

19 Kanzawa M, Semba S, Hara S, Itoh T, Yokozaki H: WNT5A is a key regulator of the epithelial-mesenchymal transition and cancer stem cell properties in human gastric carcinoma cells. Pathobiology 2013;80:235-244.

20 Heerboth S, Housman G, Leary M, Longacre M, Byler S, Lapinska K, Willbanks A, Sarkar S: EMT and tumor metastasis. Clin Transl Med 2015;4:6.

21 Wang D, Fan Z, Liu F, Zuo J: Hsa-miR-21 and Hsa-miR-29 in Tissue as Potential Diagnostic and Prognostic Biomarkers for Gastric Cancer. Cell Physiol Biochem 2015;37:1454-1462.

22 Wang QX, Zhu YQ, Zhang H, Xiao J: Altered MiRNA expression in gastric cancer: a systematic review and meta-analysis. Cell Physiol Biochem 2015;35:933-944.

23 Hou CG, Luo XY, Li G: Diagnostic and Prognostic Value of Serum MicroRNA-206 in Patients with Gastric Cancer. Cell Physiol Biochem 2016;39:1512-1520.

24 Yamamoto H, Kitadai Y, Yamamoto H, Oue N, Ohdan H, Yasui W, Kikuchi A: Laminin gamma2 mediates Wnt5a-induced invasion of gastric cancer cells. Gastroenterology 2009;137:242-252, 252 e241-246. 